\title{
Design and fabrication of compliant micromechanisms and structures with negative Poisson's ratio
}

\author{
Larsen, Ulrik Darling; Sigmund, Ole; Bouwstra, Siebe
}

Published in:

Proceedings of the Ninth Annual International Workshop on Micro Electro Mechanical Systems

Link to article, DOI:

10.1109/MEMSYS.1996.494009

Publication date:

1996

Document Version

Publisher's PDF, also known as Version of record

Link back to DTU Orbit

Citation (APA):

Larsen, U. D., Sigmund, O., \& Bouwstra, S. (1996). Design and fabrication of compliant micromechanisms and structures with negative Poisson's ratio. In Proceedings of the Ninth Annual International Workshop on Micro Electro Mechanical Systems (pp. 365-371). IEEE. https://doi.org/10.1109/MEMSYS.1996.494009

\section{General rights}

Copyright and moral rights for the publications made accessible in the public portal are retained by the authors and/or other copyright owners and it is a condition of accessing publications that users recognise and abide by the legal requirements associated with these rights.

- Users may download and print one copy of any publication from the public portal for the purpose of private study or research.

- You may not further distribute the material or use it for any profit-making activity or commercial gain

- You may freely distribute the URL identifying the publication in the public portal 


\title{
Design and Fabrication of Compliant Micromechanisms and Structures with Negative Poisson's Ratio
}

\author{
Ulrik Darling Larsen, Ole Sigmund*, Siebe Bouwstra \\ Mikroelektronik Centret (MIC), Bldg. 345E \\ *Department of Solid Mechanics, Bldg.404 \\ Technical University of Denmark \\ DK-2800 Lyngby, Denmark
}

\begin{abstract}
This paper describes a new way to design and fabricate compliant micromechanisms and material structures with negative Poisson's ratio (NPR). The design of compliant mechanisms and material structures is accomplished in an automated way using a numerical topology optimization method. The procedure allows the user to specify the elastic properties of materials or the mechanical or geometrical advantages of compliant mechanisms and returns the optimal structures. The topologies obtained by the numerical procedure require practically no interaction by the engineer before they can be transferred to the fabrication unit. Fabrication is carried out by patterning a sputtered silicon on a PECVDglass with a laser micromachining set-up. Subsequently the structures are etched into the underlying PECVDglass and the glass are underetched, all in one two-step RIE process. The components are tested using a probe placed on an xy-stage. This fast prototyping allows newly developed topologies to be fabricated and tested within the same day.
\end{abstract}

\section{INTRODUCTION}

Micromechanical devices have many promising areas of application. Some of the main areas are tools for micro- and nanofabrication, microsurgery and nano probing analysis systems. In nanofabrication the manipulation of small objects on a surface by microhandling mechanisms such as a microgripper [1] and positioning microrobotic devices [2] are steps forward towards higher functionality levels and higher flexibility. In microsurgery where accuracy is needed in microscopic and very sensitive operations, the micromechanisms may provide very precise tweezers and knifes etc. In microand nanoanalysis systems the micromechanisms can be used as positioning tools or for precision probing of a surface [3], whereas micro tweezers can be used to hold the specimens [4].

While design tools for the electronic part of MEMS are very well developed, problems such as methodical design of the mechanical parts, packaging and fabrication methods for MEMS still have to be solved. Here, we shall concentrate on design and fabrication of the mechanical parts. Because of the small dimensions, it is difficult to use ideal hinges, bearings and rigid bodies as seen in design of normal multibody systems (e.g. Erdman and Sandor [5]) for the design of MEMS. Friction and wear would cause the hinges to break down after a few operation cycles. Therefore, micromechanisms should be designed as compliant or flexible-link mechanisms. Compliant mechanisms also have the advantage that they can be built in one piece which lowers the number of fabrication steps.

Fabrication of microstructures can be done using silicon surface micromachining in thin film materials. Currently two-dimensional fabrication procedures are well developed but effort is devoted to the development of fabrication methods for two and a half [6] and fully three dimensional micromechanisms [7]. This paper will only consider two-dimensional structures. The micromachining process should be compatible with general micromachining processes to facilitate the implementation with interface electronics and actuators. Furthermore film thickness' of more than $5 \mu \mathrm{m}$ are preferred to prevent buckling and warping of the structures during testing. These demands are met by a PECVD glass developed at MIC [8]. In this paper, the structures will be operated by external probes. On-chip integrated actuation would in principle be possible using for example thermal bimorph actuators [9]. In the case of electrically conductive structures electrostatic comb drives can be applied.

\section{METHODICAL DESIGN OF COMPLIANT MECHANISMS}

Design of compliant mechanisms is often accomplished by trial and error methods. However, Howell and Midha [10] suggest a method to design compliant mechanisms by modifications of a rigid body model, Ananthasuresh et al. [11] use topology optimization methods to find the compliant mechanism 
topology which in an optimal way can perform a given manipulation task and Sigmund [12] uses a truss model and topology optimization techniques to design simple grabbing mechanisms. Topology optimization is used as a design tool when high performance and low weight of a mechanical structure is required, which is often the case in aerospace and automotive industry. This field has gained much popularity since Bendsøe and Kikuchi [13] introduced a topology optimization method using homogenization methods. For an overview of the field, the reader is referred to Bendsøe [14], and the references therein. The method presented here, allows the user to specify the mechanical or the geometrical advantages of the compliant mechanisms and the topologies obtained require only little interpretation by the engineer before they can be transferred to the fabrication unit. For design of compliant mechanisms in this paper, the topology optimization problem is defined as follows. Distribute a given amount of material within a design domain for a prescribed mechanical or geometrical advantage and such that the mechanical efficiency is maximized. The optimization problem is formulated as minimization of least squares errors in obtaining the prescribed elastic behavior and is solved using a sequential linear programming method (SLP).

\section{Computational method for compliant mechanisms}

The main difference between rigid body mechanisms and compliant mechanisms is that energy is no longer conserved between the input and the output point because of energy storage in the flexible parts of the latter. This implies that the geometrical advantage is not equal to the inverse of the mechanical advantage when we are considering compliant mechanisms. In other words: defining geometrical advantage GA as output displacement divided by input displacement and mechanical advantage MA as output force divided by input force, MA.GA will be equal to one for rigid body mechanisms with ideal hinges, whereas MA.GA will always be less than one for compliant mechanisms. One of the goals in compliant mechanism design is to get MA.GA as close to one as possible - in that way, a high mechanical efficiency of the compliant device is ensured. Typically the user wants a mechanism with some specific geometrical or mechanical advantage $G A^{*}$ or $M A^{*}$. There will also typically be some constraint on the amount of material which can be used for the structure. By choosing an upper limit $\mathrm{V}^{*}$ on the amount of material that can be distributed in the design domain, it is indirectly possible to control widths of beams in the designs.

The optimization problem can now be defined as: Distribute a given amount of material within the design domain such that the error in obtaining the prescribed values of the geometrical and mechanical advantages $G A$ and MA is minimized. The optimization problem can be written as

\begin{tabular}{rl|} 
Minimize: & $\Phi=\frac{\left(M A-M A^{*}\right)^{2}}{\left(M A^{*}\right)^{2}}+\frac{\left(G A-G A^{*}\right)^{2}}{\left(G A^{*}\right)^{2}}$ \\
subject to: & $\sum_{e=1}^{N} \rho^{e} v^{e} \leq V^{*}$ \\
and: & $0<\rho_{\min } \leq \rho^{e} \leq 1, \quad e=1, \ldots, N$
\end{tabular}

where $N$ is the number of elements or design variables, $\rho$ $\mathrm{e}$ is the density of material in element $e$ (design variable e), $\rho_{\min }$ is the lower side constraint on the design variables (for computational reasons) and $v^{e}$ is the volume of element $e$. Typically the algorithm requires several hundred iterations to converge, each iteration step requiring a finite element analysis with two load cases (solving (1)), a simple analytical sensitivity analysis and a design update using the Simplex algorithm. For more details on the computational algorithms and methods, the reader is referred to the general literature on topology optimization (e.g. Bendsøe [14] and Sigmund [15]).

\section{Compliant mechanism design examples}

Figure 1 shows the design domains and input and output load cases for five considered compliant mechanism design problems.

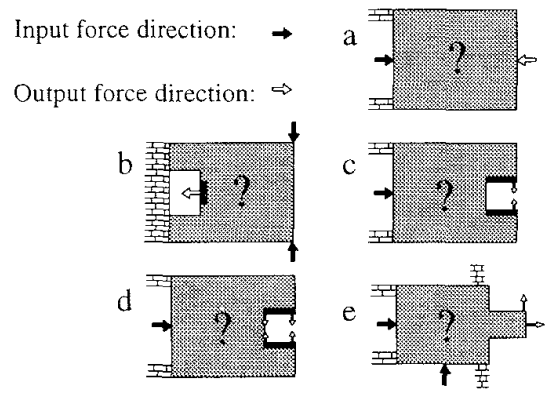

Figure 1. Design domains and input and output load cases for five compliant mechanisms. The gray areas define the design domains whereas white and black areas define areas which are specified to be void or to consist of solid material, respectively. Black arrows denote point and direction of the input force and white arrows denote points and directions of the prescribed output forces or displacements. Numbers denotes the magnitude of the forces.

The rectangular design domains are discretized by typically 3000 quadrilateral finite elements where the density of material in each element represents one design variable. The resulting designs are shown in figure 2. By using penalization of gray areas in the design algorithm, the amount of gray areas are minimized but still their appearance can not be fully eliminated. 


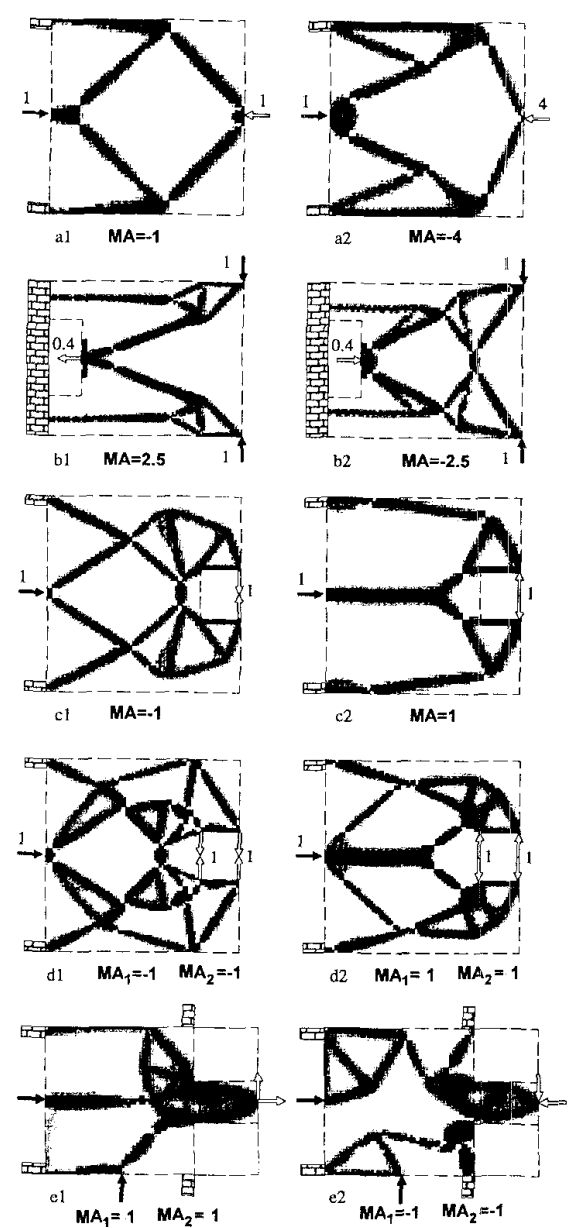

Figure 2. Ten optimized compliant micromechanisms. White colors indicate void areas, black colors indicate solid areas and gray areas indicate areas of medium density. Numbers denote the size of the forces.

The five mechanisms can be characterized as follows. Mechanism $a$ is a force inverter which converts a horizontal input force to a force in the opposite direction at the output point. The mechanical or geometrical advantages can be prescribed such that force or displacement amplification can be achieved. Figure 2. $a 1$ and 2.a2 show $\mathrm{a} 1: 1$ and a $4: 1$ force inverter respectively. The mechanism in figure $2 . a 2$ can also be seen as a displacement amplifier: An input displacement (black arrow) is converted to a four times bigger output displacement in the opposite direction. It should be noted that the design procedure only considers linear displacements and therefore the geometrical advantages only hold for small input displacements. Figure $2 . b 1$ and $2 . b 2$ show the resulting designs for a cleaving mechanism (this design example was used in reference [11]). There are two input forces which should be converted into a compressive or a tensile force at the output piston (which is prescribed to be solid as seen in figure $1 \mathrm{~b}$ ). Mechanisms $c$ and $d$ represent the design of gripping mechanisms. For grippers $c 1$ and $c 2$, the output points are the outer points of the jaws which are specified to close and open, subject to the horizontal input force, respectively. For grippers $d 1$ and $d 2$, the jaws are specified to move in parallel. This was done by specifying two separate output load cases and the design problem was therefore extended to include two prescribed geometrical advantages and two prescribed mechanical advantages. Finally, mechanism $e$ represents the design of a micropositioning device. Given a design domain and two input actuators, the problem is to find the mechanism topology which can make the output point to be controlled independently in the vertical and the horizontal directions. Figure 2.e 1 shows a design where a horizontal input force (mid left edge) results in a horizontal movement of the output point and a vertical input force (mid lower side) results in a vertical movement of the output point. If we want to use comb drives as actuators, we can only get attracting input forces. Fortunately, the method is able to produce the micropositioning device seen in figure 2.e2; This mechanism has the opposite output behavior compared to $e 1$. All these mechanisms can also be used in the opposite direction where a force or a displacement at the output gives an associated force or displacement at the input.

\section{DESIGN OF STRUCTURES WITH NEGATIVE POISSON'S RATIO}

Design of materials with a special kind of elastic properties has been the goal of many researchers recently. The existence of materials with negative Poisson's ratios (NPR), or in other words, the existence of materials that expand transversely subject to an applied tensile load has been questioned by researchers and engineers for a long time. However, thermodynamics allows the Poisson's ratio of an isotropic material to approach -1. Among other applications (see e.g. Lakes [9]), materials with negative Poisson's ratio can advantageously be used in the design of hydrophones [16] and other sensors. One reason is, that the low bulk modulus of these materials make them more sensitive to hydrostatic pressure. As the bulk modulus of an isotropic material is defined as $K=E /(3(1-2 v))$, where $E$ is Young's modulus and $v$ is Poisson's ratio, the sensitivity to hydrostatic pressure is increased by almost one order of magnitude for a Poisson's ratio -1 material compared to a material with an ordinary Poisson's ratio 0.3 . A theoretical material microstructure with Poisson's ratio -1 was first reported by Almgren [17]. A practical material with negative 
Poisson's ratio was first developed by Lakes [18], who treated an open walled foam material with heat and pressure to obtain the negative Poisson's ratio effect. Lake's foams have average cell sizes down to 0.3 millimeter and Poisson's ratios down to -0.8. In Sigmund [19][20] and [12], a numerically based method is used to design materials with any thermodynamically admissible elastic properties. In this paper, we will show that it is possible to use microstructures in the design of $2 \mathrm{D}$ isotropic material with negative Poisson's ratios and cell sizes down to $50 \mu \mathrm{m}$ using topology optimization. These microstructures are referred to as NPR-materials and can be fabricated by utilizing silicon surface micromachining techniques.

It might be objected that the NPR-materials produced in this work are structures rather than materials. However, knowing that every material has a structure if one looks at it at a sufficiently small scale, the distinction between "materials" and "structures" is blurred. Defining materials as repeated structures which can not be seen by the naked eye, the negative Poisson's structure produced in this paper are materials indeed, and they are comparable to the naturally existing material cork (zero Poisson's ration), which has a microstructure on the same length scale.

For the design of NPR-materials we will consider periodic microstructures where the smallest repetitive unit called the base cell will be the design domain and the design goal is to minimize the error in obtaining the prescribed elastic properties for a fixed amount of material in the base cell. The behavior of both mechanisms and NPR-materials is analyzed by discretizing the design domain by four node quadrilateral finite elements and solving the finite element problems for several loading cases. The design variables are given as the density of material in each finite element. As in the previous section the optimization problems are solved using the sequential linear programming method (SLP).

\section{Computational method}

The behavior of a linear elastic material follows the generalized Hooke's law

$$
\sigma_{i j}=E_{i j k l} \varepsilon_{k l}
$$

where $E_{i j k l}$ is the elasticity tensor, $\sigma_{i j}$ and $\varepsilon_{i j}$ are the stress and strain tensors. By distributing a prescribed amount of material within the design domain (the base cell), we can design a porous and periodic structure with the prescribed elasticity tensor $\mathrm{E}_{\mathrm{ijk}}^{*}$, assuming plane stress conditions and with prescribed density $\rho_{\text {cell }}^{*}$. Again, the optimization problem is formulated as a least squares problem.

$$
\begin{aligned}
\text { Minimize: } & \Phi=\left(E_{i j k l}^{H}-E_{i j k l}^{*}\right)^{2} \\
\text { subject to: } & \sum_{e=1}^{N} \rho^{e} v^{e} \leq \rho_{\text {cell }}^{*} \\
\text { and: } & 0<\rho_{\min } \leq \rho^{e} \leq 1, \quad e=1, \ldots, N
\end{aligned}
$$

where as before, $N$ is the number of finite elements or design variables, $\rho^{e}$ is the density of material in element $e$ (design variable $e$ ), $\rho_{\min }$ is the lower side constraint on the design variables (for computational reasons) and $v^{e}$ is the volume of element $e . E_{i j k l}^{H}$ denotes the homogenized or averaged elasticity tensor for the inhomogeneous material, which can be found using the standard homogenization method (e.g. Bourgat [21]). The homogenization method implies the solving of a finite element problem with three load cases, namely a horizontal pull, a vertical pull and a shear pulling case, just as one would do to test a real material.

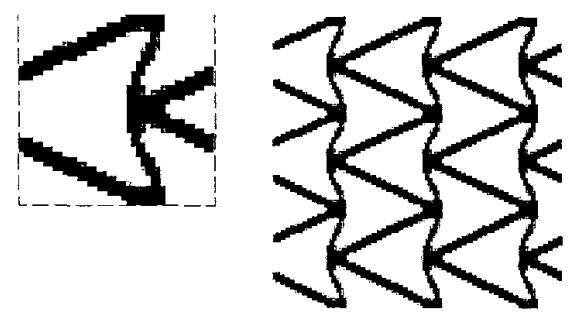

Figure 3. Material with Poisson's ratio - 0.8 obtained from a ground structure with 40 by 40 elements and vertical symmetry enforcement.

\section{NPR-material design examples.}

Design of an NPR-material was done by specifying the elastic properties of a material with Poisson's ratio 0.8 and solving the optimization problem (3) for a quadratic base cell discretized by 1600 quadrilateral finite elements each representing one design variable. The resulting topology is seen in figure 3 (left). The base cell is repeated in figure 3 (right), where the mechanism is seen more clearly. When the material is compressed horizontally, the triangles will collapse and result in a vertical contraction which is the characteristic behavior of a negative Poisson's ratio material.

\section{FABRICATION OF MICROSTRUCTURES}

The achieved designs for compliant micromechanisms and NPR-materials were fabricated using silicon surface micromachining. In stead of conventional photolithography, we applied a direct writing approach using laser micromachining of a silicon layer sputter deposited on top of a glass layer. To manufacture the mechanisms and NPR-materials we need to create an 
input file containing the mask patterns for the laser micromachining. The mask is a "negative" of the structure as seen in figure 4 since the dark areas denote the areas to be removed by the laser. The numerically produced topologies should be slightly postprocessed by the operator, however they are easily interpreted as frame structures. The only points of caution are the grey regions where the topologies are not clearly defined. Some of the postprocessed mechanisms are shown in figure 4.
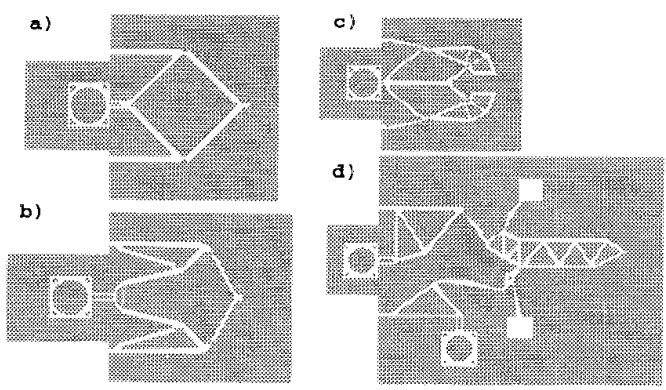

Figure 4. Four postprocessed micromechanisms. a) an I:I inverter, b) an 1:4 inverter, c) a gripper and d) a positioner. These designs are now ready to be transfered to the laser micromachining set-up.

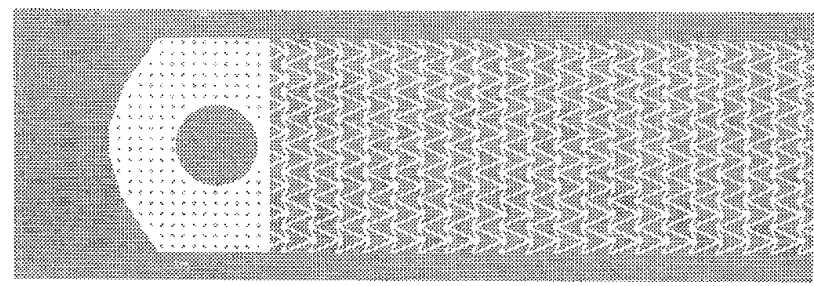

Figure 5. Interpretation of one of the NPR-material designs.

To be able to mechanically test the negative Poisson's ratio material, the base cell is repeated 8 and 20 times in the two directions. The resulting bar is clamped at one end and equipped with a handle at the other end as seen in figure 5 .

The structures were realized in an isotropic $6 \mu \mathrm{m}$ thick germanium doped PECVD-oxinitride that was developed at the Microelectronics Centre [8]. This type of material as device layer, enables us to make thick films $(>5 \mu \mathrm{m})$ without grain boundaries and with very low stress and isotropic elastic properties. The PECVD-glass was deposited on a $4 ",\langle 100\rangle$ silicon wafer and subsequently thermally annealed at $800^{\circ} \mathrm{C}$ in $\mathrm{N}_{2}$ for 1.7 hours to bring the compressive stress in the glass close to zero.

The choice of a PECVD-glass enables us to use a simple two-step RIE process to make suspended structures. A $2 \mu \mathrm{m}$ thick film boron doped silicon was sputtered on top of the glass as a masking layer (fig.6a). The mechanism designs were etched into the sputtered silicon by direct etching with a laser micromachining system (fig.6b) [6],[22]. This method of rapid prototyping made it possible to test a large amount of device designs as they were completed.

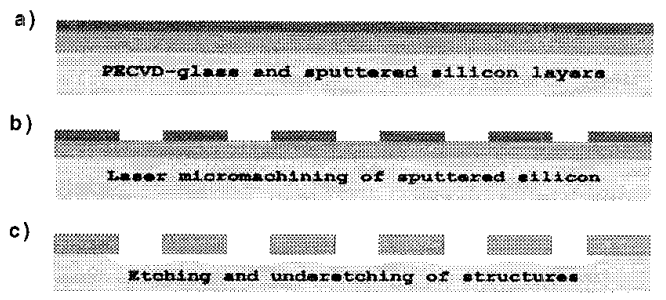

Figure 6. Steps in the process sequence: a) After deposition of $6 \mu \mathrm{m}$ PECVD-glass and $2 \mu \mathrm{m}$ silicon. b) After laser micromachining of a prototype mask. c) After two-step RIE process: Anisotropic glass etch followed by an isotropic silicon etch.

The spot shape of the $1 \mu \mathrm{m}$ laser beam results in a roughness of the sidewalls as seen in figure 7 (lower left corner). The subsequent two-step RIE process includes a $6 \mu \mathrm{m}$ anisotropic glass etch in CF4/CHF3 and a $20 \mu \mathrm{m}$ isotropic silicon etch in SF6 at 120 mTorr.

In figure 7 (left) the final result of a suspended NPRmaterial beam can be seen. In the upper right of figure 7 (left) the cross section of the beam shows the isotropy of the RIE-etch, the selectivity is approximately 1:2. Figure 7 (right) shows 3 compliant mechanism prototypes.

\section{EXPERIMENTAL RESULTS}

The samples with the micromechanisms was placed on a chuck with a vacuum fixture (fig.8). To operate the structures a probe on a vertically adjustable arm was placed on an $x-y$-stage. The stage was manually operated and the movements were measured on a scale with 0.5 $\mu \mathrm{m}$ resolution. The structures can be monitored on a screen using a video camera and the response of the mechanisms was recorded on a video tape for subsequent evaluation.

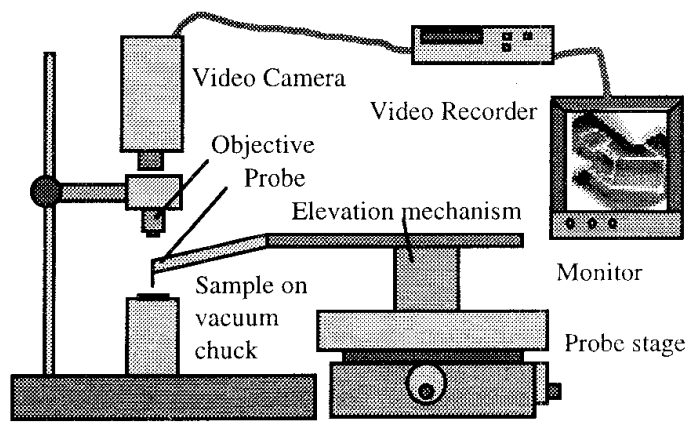

Figure 8. Test setup. The sample are placed on a chuck and the mechanisms are tested by a probe on a stage. The movements are recorded. The experiments are evaluated using a monitor. 
For the NPR-materials the sideways extension were determined by adding the two sideways displacements of the two sides of the cantilever. The Poisson's ration can be found from:

$$
v=\frac{-\Delta b / b}{\Delta l / l}
$$

where $\Delta b$ is the sideways extension, $\Delta l$ is the axial elongation, $b$ is the width and $l$ is the length of the beam. In the practical test, the NPR-materials designed for a Poisson's ratio of -0.8 were measured to have a NPR of $0.92 \pm 13 \%$ up to a $1.6 \%$ elongation (see figure $9 \mathrm{~A}$ ).

Output displacements were determined using a ruler on the screen after calibration. The accuracy is determined by the resolution which amounts to $1 \mu \mathrm{m}$. The graph in figure 9B presents the response of the 1:1 and 1:4 displacement inverters. They both show good linearity until the moment where buckling appears. In the linear regions the response of the $1: 1$ and the $1: 4$ displacement inverters are $1: 1 \pm 10 \%$ and $1: 3.6 \pm 10 \%$ respectively. Eventually the structures fracture.

\section{Discussion}

In practice it shows that one should be careful with 2-dimensional calculations when the width of the interconnections becomes larger than the thickness of the structure layer. To reach better results the structures should be fabricated using thicker layers, for instance by using electroplated materials.

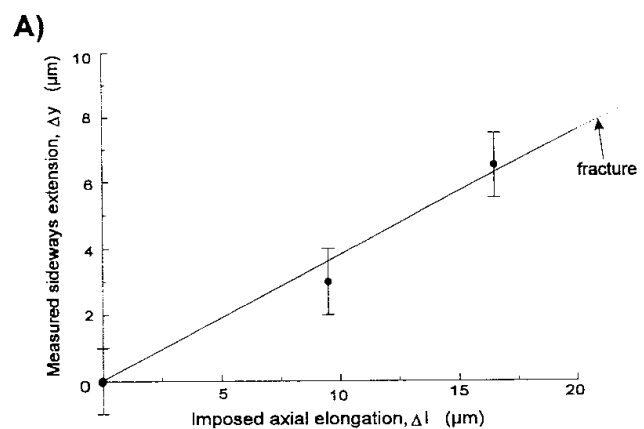

B)

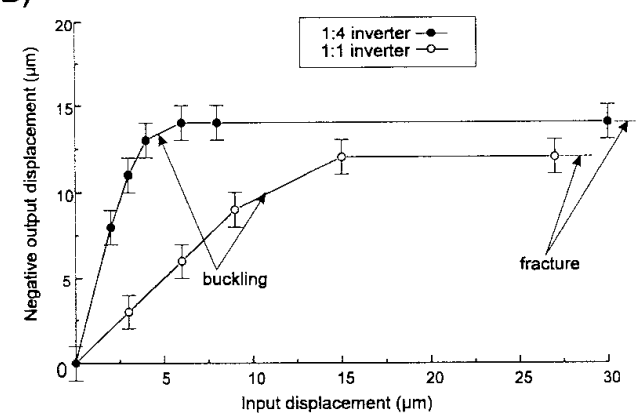

Figure 9. A: Response of an NPR-material. Measured sideways extension vs. imposed axial elongation. The resulting NPR measured was $0.92+13 \%$ B: Measured response of $1: 1$ and $1: 4$ displacement inverters. The linear displacement transformation holds until buckling appears and eventually the structures fracture. The resulting linear displacement inversions measured were 1:1 $+1-10 \%$ and 1:3.6+1-10\%, respectively.

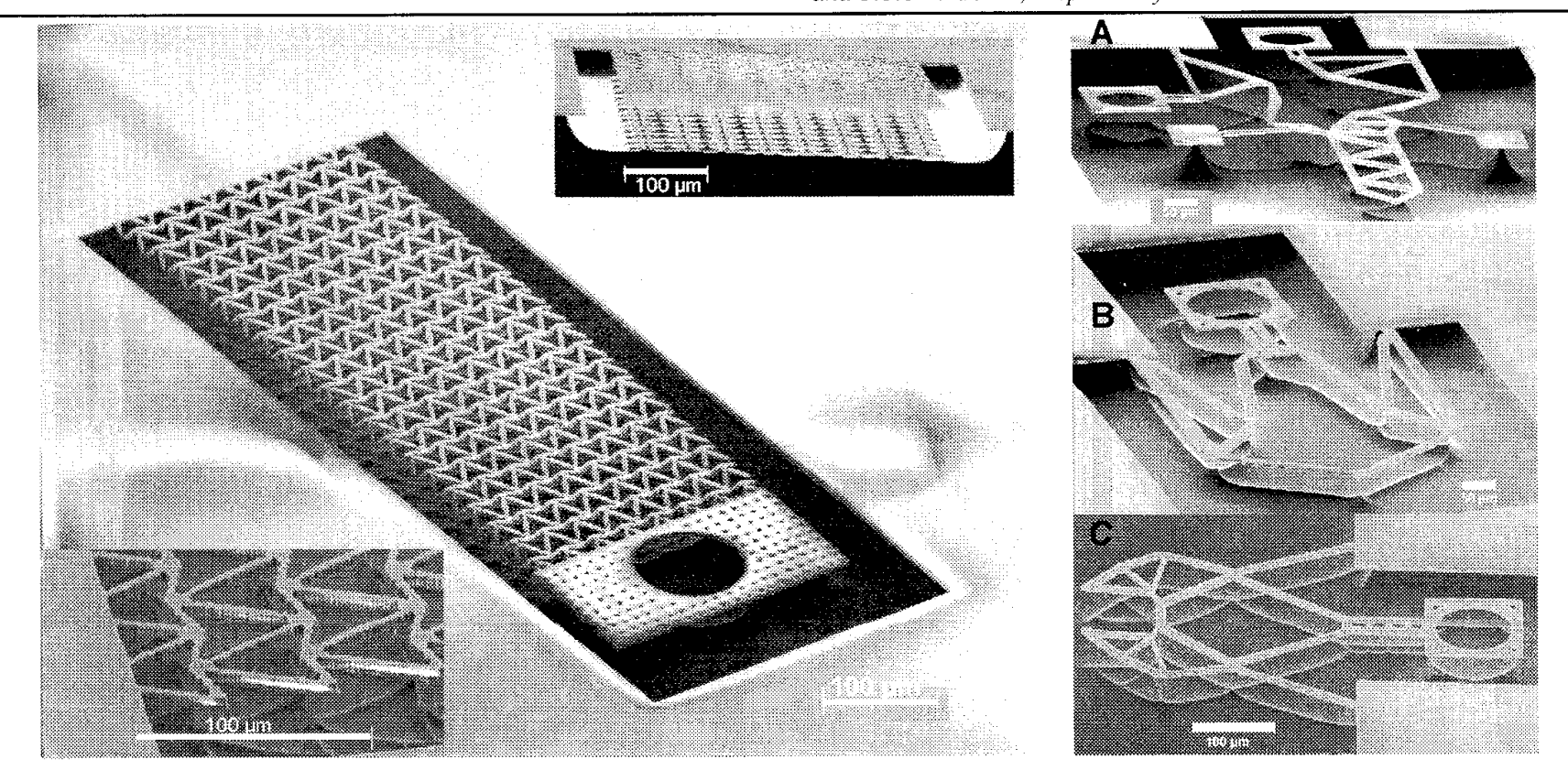

Figure 7. Left: NPR-material testing bar composed of 20 by 8 unit cells. The handle is seen in the front. Upper right: View of the cantilever in the axial direction, after cleaving. The pattern of the beam is also present at the bottom of the recess. Lower left: Close-up of the NPRmaterial. The roughness of the sidewalls is due to the finite resolution of the laser micromachining.

Right: Compliant micromechanisms: a) a positioner mechanism. b) a 4:1 inverter mechanism c) a gripper mechanism. 
Requirements could also be reached by scaling down the mechanisms, but this would require higher resolution fabrication methods. Elongations larger than $2 \%$ could not be reached because the structures fracture due to stress concentrations in the fragile glass material. More enduring structures would be obtained with thicker films or by using more elastic and less brittle materials such as polymers or electroplated copper or nickell which require alternative fabrication techniques. Non-linearities such as those due to buckling could be avoided by increasing the widths of buckling-prone elements in the structure.

\section{CONCLUSION}

Fabrication of compliant micromechanisms have the advantage that they can be built in one piece with very few fabrication steps. Compliant micromechanisms and materials with negative Poisson's ratio have been designed using a computational design tool to generate topology optimized structures. The method allows the user to specify the mechanical or the geometrical advantages of the compliant mechanisms and the resulting topologies are easily interpreted by the engineer. The generated mechanical structures and NPRmaterials are fabricated in PECVD oxinitride by etching a pattern into a masking layer with a laser micromachining set-up and subsequently etching and underetching the oxinitride in two RIE processes. Structures for force or displacement amplifiers, attenuators and inverters and materials with negative Poisson's ratio can easily be designed and fabricated. Design, fabrication and characterization can be done within one/two days, leading to fast prototyping.

\section{ACKNOWLEDGMENT}

The authors would like to thank Matthias Müllenborn for his assistance with the laser micromachining, Torben Storgaard-Larsen and Leif S. Johansen for useful information on processing and the laboratory staff for assistance with processing in the cleanroom.

The work presented in this paper received financial support from Denmark's Technical Research Council (Programme of Research on Computer-Aided Design).

\section{REFERENCES}

[1] G. Thornell, M. Bexell, J-Å. Schweitz, S. Johansson, The Design and Fabrication of a Gripping Tool for Micromanipulation, Digest of Techn. Papers Transducers '95, June 1995, vol,2 388-391.

[2] J. F. L. Goosen, R. F. Wolfenbuttel, Object Positioning Using a Surface Micromachined Distributed System, Digest of Techn. Papers Transducers '95, June 1995, vol.2 396-399.
[3] P.-F. Indermuehle, C. Linder, J. Brugger, V. P. Jaecklin and N.F. de Rooij, Design and fabrication of an overhanging $x y-$ microactuator with integrated tip for scanning surface profiling", Sensors \& Actuators A, 43 (1994) 346-350.

[4] C. G. Keller, R. T. Howe, Nickel-Filled Hexsil Thermally Actuated Tweezers, Digest of Techn. Papers Transducers '95, June 1995, vol.2 376-379

[5] A. G. Erdman, G. N. Sandor, Mechanism Design, Analysis and Synthesis, Volume 1, Prentice Hall, London (1991).

[6] M. Müllenborn, H. Dirac, J. W. Petersen, S. Bouwstra, Fast 3D Laser Micromachining of Silicon for Micromechanical and microfluidic applications, Digest of Techn. Papers Transducers '95, June 1995, vol.1 166-169.

[7] A.-L. Tiensuu, M. Bexell, J.-Å. Schweitz, L. Smith, S. Johansson, "Assembling three-dimensional microstructures using gold-silicon eutectic bonding", Sensors \& Actuators, A 45 (1994) 227 236

[8] T. Storgaard-Larsen, S. Bouwstra, O. Leistiko, Opto-Mechanical Accelerometer Based on Strain Sensing by a Bragg Grating in a Planar Waveguide, Digest of Techn. Papers Transducers 95, June 1995, vol.2 667-670.

[9] W. Riethmüller, W. Benecke, Thermally Excited Silicon Microactuators, IEEE Transactions Electron Devices, ED-35(6) (1988) 758

[10] L. L. Howell, A. Midha, A method for the design of Compliant Mechanisms With Small length Flexural Pivots, Transactions of the ASME, 116 (1994) 280-290.

[11] G. K. Ananthasuresh, S. Kota, Y. Gianchandani, A Methodical Approach to the Design of Compliant Micromechanisms, Solid-State Sensor and Actuator Workshop, June 1994, 189-192.

[12] O. Sigmund, Some inverse problems in topology design of materials and mechanisms. Proc. of the IUTAM Symposium on Optimization of Mechanical Systems, Stuttgart, Germany, March 2631 (1995), (Ed. W. Schielen), Kluwer (to appear) also as DCAMM Report No. 499, April 1995.

[13] M. P. Bendsøe, N. Kikuchi, Generating optimal topologies in structural design using a homogenization method, Comp. Meth. Appl. Mechs. Engng. , 71 (1988) 197-224.

[14] M. P. Bendsøe, Methods for the Optimization of Structural Topology, Shape and Material, Springer Verlag, Berlin, 1995.

[15] O. Sigmund, Design of Compliant Mechanisms using Topology Optimization. Manuscript under preparation (1995)

[16] M. Avellanada, P. J. Swart, Calculating the performance of 1-3 piezo composites for hydrophone applications : an effective medeum approach, To appear, (1995)

[17] R. F. Almgren, An isotropic three-dimensional structure with Poisson's ratio $=-1$, J. Elasticity, 15 (1985) 427-430.

[18] R. Lakes, Foam Structures with Negative Poisson's Ratio. Science 235 (Feb. 1987) 1038.

[19] O. Sigmund, Materials with prescribed constitutive parameters: an inverse homogenization problem, Int. J. Solids Struct., 31 (17), (1994) 2313-2329.

[20] O. Sigmund, Design of Material Structures using Topology Optimization. DCAMM Special Report No. 69 (1994), Ph.D.thesis, The Danish Center for Applied Mathematics and Mechanics, Technical University of Denmark, DK-2800 Lyngby, December 1994. [21] J. F. Bourgat, Numerical experiments of the homogenization method for operators with periodic coefficients, Lecture Notes in Mathematics 704, Springer Verlag, Berlin, (1977) 330-356.

[22] M. Müllenborn, M. Heschel, H. Dirac, S. Bouwstra, Laser direct writing of silicon oxide for rapid prototyping, procs. Micromechanics Europe 95 Workshop, September 1995, pp. 64-67. (Paper also accepted for publication in J. Micromechanics and Microengineering). 